\title{
Tumour stage on re-staging transurethral resection predicts recurrence and progression-free survival of patients with high-risk non-muscle invasive bladder cancer
}

\author{
Mohamed Bishr, MD; ${ }^{*}$ Jean-Baptiste Lattouf, MD, FRCSC; ${ }^{*}$ Mathieu Latour, MD, FRCPC; ${ }^{\dagger}$ Fred Saad, MD, FRCSC
}

*Department of Urology, Centre hospitalier de l'Université de Montréal, Montreal, QC; 'Department of Pathology, Centre hospitalier de l'Université de Montréal, Montreal, QC

Cite as: Can Urol Assoc J 2014;8(5-6):e306-10. http://dx.doi.org/10.5489/cuaj.1514 Published online May 21, 2014.

\section{Abstract}

Introduction: To identify patients who should be considered for early radical cystectomy, we evaluated the clinical and pathological variables affecting the outcome of patients with high-risk nonmuscle invasive bladder cancer (NMIBC) who underwent re-staging transurethral resection (re-TUR).

Methods: We reviewed the clinical data of 453 patients treated for urothelial carcinoma between 2006 and 2010. In total, 94 patients underwent re-TUR after their initial TUR. Of these, 72 were not upstaged to muscle invasive disease and were therefore included in our study.

Results: On re-TUR, 31 patients had no residual tumour (T0) and 41 patients had residual NMIBC. A statistically significant difference was noted between patients with pT0 and patients with residual NMIBC on re-TUR in regard to tumour recurrence and progression (39\% vs. $83 \%, p<0.001)(6 \%$ vs. $34 \%, p=0.005)$, respectively. On multivariate analysis, tumour stage on re-TUR and the regimen of intravesical bacillus Calmette-Guérin (BCG) therapy (induction vs. maintenance) remained independent predicting factors for recurrence-free survival (RFS) ( $p=0.001$, hazard ratio [HR]: 1.77), $(p<0.001 \mathrm{HR}: 0.16)$ and progression-free survival (PFS) $(p=0.014$, HR: 2.11), ( $p=0.008$, HR: 0.097), respectively.

Conclusions: The presence of T0 on re-TUR is associated with better RFS and PFS and could be a predictive factor for candidates for conservative management. Patients with persistent NMIBC on reTUR require close follow-up and, in some cases, could be considered for early cystectomy. Maintenance intravesical BCG therapy can improve RFS and PFS in patients with high-risk NMIBC. This study is limited by its retrospective nature and the relatively small number of patients in the cohort.

\section{Introduction}

Bladder cancer is the second most common urologic malignancy. About $70 \%$ of bladder tumours are non-muscle invasive at presentation. Of these, $70 \%$ are confined to the blad- der mucosa (Ta), 25\% are invading the lamina propria (T1) and $5 \%$ are carcinoma in situ $(\mathrm{CIS}){ }^{1}$

The optimal management of non-muscle invasive bladder cancer (NMIBC) is based on 3 criteria: (1) complete transurethral resection (TUR), (2) effective intravesical treatment in association with early detection of conservative treatment failure, and (3) timely intervention in the form of radical cystectomy. ${ }^{2}$ Re-staging TUR (re-TUR) to manage high-risk NMIBC is now widely accepted because it improves clinical staging, detects more tumours than initial TUR and reduces the rate of early recurrence. ${ }^{3}$

Recent data support the concept that performing radical cystectomy in patients with high-risk NMIBC before, rather than after, progression to muscle-invasive tumour improves survival. Thus, an approach using reliable risk stratification to identify candidates at high risk for progression and candidates for early cystectomy is required. ${ }^{2}$ Since the role of biological markers is not well-defined, it has been suggested that risk stratification should be based on clinical and pathological features. ${ }^{4}$

Adverse prognostic features that have been associated with a high risk of progression include number of tumours ( $>3$ lesions), tumour size $(>3 \mathrm{~cm})$, hydronephrosis, invasion of the lamina propria (T1), depth of sub-mucosal invasion (T1a vs. T1b), high-grade, concomitant CIS, T1 disease on re-TUR, tumour recurrence on first follow-up cystoscopy, T1 recurrence and superficial prostatic urethral recurrence..$^{5-10}$

To identify patients who should be considered for early radical cystectomy, we evaluated the clinical and pathological variables that may affect the outcome of patients with high-risk NMIBC who underwent re-TUR. Outcome was defined in terms of recurrence-free survival (RFS) and progression-free survival (PFS).

\section{Methods}

We reviewed the clinical data of 453 patients with bladder urothelial carcinoma treated between 2006 and 2010 to identify patients with newly diagnosed NMIBC who underwent re-TUR. Of these, 94 patients underwent re-TUR within 
3 months after their initial TUR due to either incomplete resection, absence of muscularis propria, or second-look TUR. Depending on the clinical and pathological adverse prognostic features, intravesical chemotherapy and/or bacillus Calmette-Guérin (BCG) therapy were administered after re-TUR. Intravesical chemotherapy (mitomycin, doxorubicin, or epirubicin) used in $37 \%$ of patients, was in the form of a single intravesical instillation within the 24-hour postre-TUR period. Generally immediate postoperative chemotherapy was given in patients where complete resection appeared to have been performed and BCG was not planned. BCG therapy was either as a conventional 6-week induction course alone or induction course followed by maintenance therapy, which entailed 3 instillations at 3 months post-reTUR, then semi-annually thereafter for 3 years.

Patients were followed with cystoscopy and urine cytology every 3 to 4 months in the first year, then every 4 to 6 months for the following 3 years and then annually. In case of tumour recurrence, patients either underwent TUR followed by intravesical BCG therapy or radical cystectomy according to their clinical and pathological risk factors.

The end points of the study were RFS and PFS. Tumour recurrence was defined as the presence of any tumour during follow-up cystoscopy. Tumour progression was defined as progression to muscle invasive disease. Patients who underwent radical cystectomy for NMIBC and were not upstaged to muscle invasive disease were censored because they were no longer at risk of local progression.

Associations between categorical variables and tumour recurrence or progression were determined by the Chisquare test. Differences in variables with a continuous distribution across dichotomous categories were assessed using the Mann-Whitney test. RFS and PFS were calculated from the time of re-TUR using Kaplan-Meier method and compared with the log-rank test. Multivariate analyses were done using the Cox proportional hazards regression model to determine independent predictive factors of RFS and PFS. All reported $p$ values were two-sided, and statistical significance was set at $<0.05$. All statistical tests were done using SPSS v.16.0 (SAS Institute, Cary, NC).

\section{Results}

Of the 94 patients who underwent re-TUR, 29 had Ta tumours (8: Ta low grade, 21: Ta high grade) and 65 had T1 tumours (1: T1 low grade, 64: T1 high grade) on their initial TUR. On re-TUR, 52\% of patients (49/94) had residual NMIBC and 14\% (13/94) were upstaged to muscle invasive disease. The incidence of upstaging to muscle invasive disease on re-TUR was higher in T1 tumours than Ta tumours $(17 \%$ vs. $7 \%, p=0.193)$, while the incidence of T0 (no residual tumour) on re-TUR was higher in Ta tumours than in $\mathrm{T} 1$ tumours ( $48 \%$ vs. $28 \%, p=0.052$ ).
Patients whose tumours were upstaged to muscle invasive disease on re-TUR $(n=13)$ or whose follow-up data were incomplete $(n=9)$ were excluded from further analysis.

Our study cohort consisted of 72 patients, including 56 males and 16 females, with median age 67 years (Table 1). On initial TUR, 25 patients had Ta (7: Ta low grade, 18: Ta high grade) and 47 had T1 high-grade tumours. Ninety percent of patients had high-grade tumours and $8 \%$ had concomitant CIS. The median follow-up period was 32.5 months. Twenty-seven patients out of the 72 (37\%) received a single instillation of intravesical chemotherapy within 24 hours post-re-TUR. In total, 60 patients (83\%) received intravesical BCG therapy either as an induction course alone $(n=33)$ or maintenance course $(n=27)$. Twelve patients received more than 1 course of BCG and 14 (2: induction course alone, 12: maintenance course) did not complete their BCG course due to adverse effects or intolerance.

Tumour recurrence occurred in $64 \%$ of patients (46/72) and it was associated with multifocality of the tumour on initial TUR $(p=0.005)$, higher T stage on re-TUR $(p=0.001)$, and regimen of intravesical BCG therapy (induction alone vs. maintenance) $(p<0.001)$. On the other hand, tumour progression to muscle invasive disease occurred in $22 \%$ of patients (16/72) and was associated with hydronephrosis $(p=0.005)$, higher T stage on initial TUR $(p=0.034)$, higher T stage on re-TUR ( $p=0.005)$, and regimen of intravesical BCG therapy (induction alone vs. maintenance) $(p=0.015)$. A statistically significant difference was noted between patients with T0 on re-TUR and patients with residual NMIBC on re-TUR in regard to tumour recurrence and progression $(39 \%$ vs. $83 \%, p<0.001)(6 \%$ vs. $34 \%, p=$ $0.005)$, respectively (Table 2 ).

The overall median RFS was 13 months (95\% confidence interval $\mathrm{Cl}$, 8-19) while overall median PFS was not reached (mean: 57 months; 95\% Cl 50-63). On univariate survival analysis using the Kaplan-Meier method and log-rank test for comparison, a shorter RFS was found in patients with multiple tumours on initial TUR $(p=0.008$, log-rank $=6.928)$, hydronephrosis $(p=0.035$, log-rank= 4.454), higher $\mathrm{T}$ stage on re-TUR $(p<0.001$, log-rank $=25.717)$, no early intravesical chemotherapy versus early intravesical chemotherapy ( $p=0.035$, log-rank $=4.461)$ and induction BCG vs. maintenance BCG therapy $(p<0.001, \log$-rank $=20.885)$ (Fig. 1, part B). Likewise, a shorter PFS was found in patients with hydronephrosis $(p=0.007$, log-rank $=7.269)$, higher $\mathrm{T}$ stage on initial TUR $(p=0.032$, log-rank $=4.607)$, higher T stage on re-TUR ( $p=0.001$, log-rank $=17.1)$ and induction BCG versus maintenance BCG therapy ( $p=0.009$, log-rank = 6.740) (Fig. 1, part D).

We also noted that patients with pT0 on re-TUR had better RFS and PFS compared to patients with residual NMIBC on re-TUR $(p<0.0001$, log-rank $=21.545)(p=0.004$, log- 
Bishr et al.

\begin{tabular}{lc}
\hline Table 1. Patients characteristics & \\
\hline Median age (range) & $\mathbf{6 7}$ years (48-88) \\
\hline Gender & $56(78 \%)$ \\
Male & $16(22 \%)$ \\
Female & \\
Pathology of initial TUR & $7(10 \%)$ \\
Ta low grade & $18(25 \%)$ \\
Ta high grade & $47(65 \%)$ \\
T1 high grade & \\
Size of tumour & $44(61 \%)$ \\
$<3$ cm & $28(49 \%)$ \\
$>3$ cm & \\
Multifocal tumour & $34(47 \%)$ \\
Yes & $38(53 \%)$ \\
No & \\
Concomitant CIS & $6(8 \%)$ \\
Yes & $66(92 \%)$ \\
No & \\
Regimen of intravesical BCG therapy & 32.5 months $(4-72)$ \\
Induction alone & $27(55 \%)$ \\
Maintenance & \\
Early intravesical chemotherapy & \\
Yes & \\
No & \\
Median follow-up period (range) & \\
\hline TUR: transurethral resection; CIS: carcinoma in situ; BCG: bacillus Calmette-Guérin. \\
\hline
\end{tabular}

rank $=8.337$ ), respectively (Fig. 1 , parts $A$ and $C$ ).

On multivariate analysis of predictive factors for RFS and PFS using the Cox proportional hazards regression model, only $T$ stage on re-TUR and regimen of intra-vesical BCG therapy remained independent prognostic factors for RFS ( $p$ $=0.001$, hazard ratio [HR]: 1.776), $(p<0.001, \mathrm{HR}: 0.165)$, respectively and PFS $(p=0.014, \mathrm{HR}: 2.114),(p=0.008$, HR: 0.097), respectively (Table 3 ).

\section{Discussion}

The management of high-risk NMIBC remains a subject of controversy. Although several attempts have been made to identify patients at high risk for progression who should undergo early cystectomy, the question as to when to switch from conservative therapy to radical cystectomy remains largely unanswered. In the present retrospective study, we evaluated the clinical and pathological variables that affect RFS and PFS in patients with high-risk NMIBC who underwent re-TUR to help identify candidates for either conservative management or radical cystectomy.

In the study cohort, re-TUR detected residual NMIBC in $52 \%$ of patients and improved clinical staging in $14 \%$ of patients whose tumours were upstaged to muscle invasive disease. The incidence of residual tumours on re-TUR was higher in patients with $\mathrm{T} 1$ than in patients with $\mathrm{Ta}(p=0.05)$.

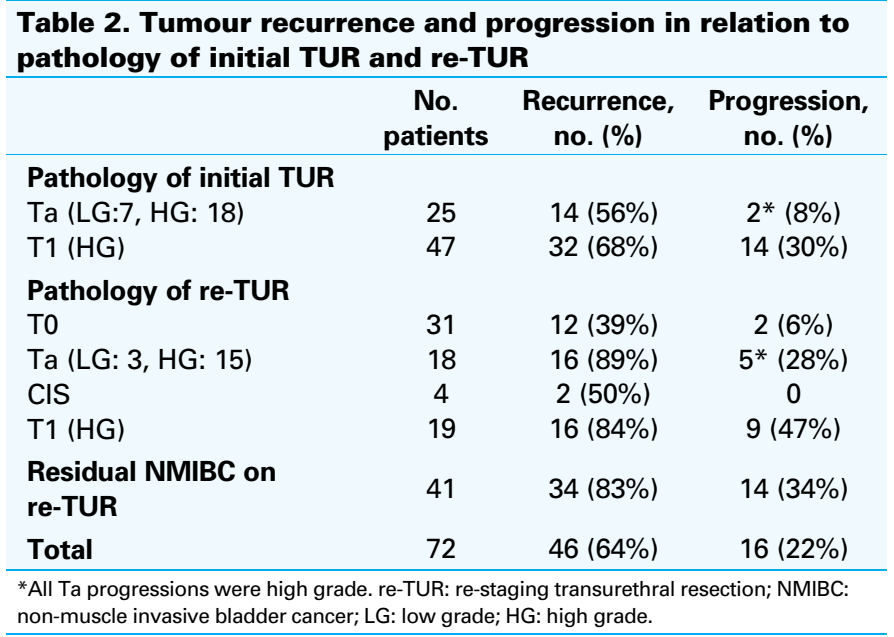

Likewise, the incidence of tumour upstaging in patients with $\mathrm{T} 1$ was higher than in patients with $\mathrm{Ta}(p=0.19)$. These rates of residual tumour and upstaging on re-TUR are within the range reported in literature $26 \%$ to $83 \%$ and $9 \%$ to $49 \%$ respectively; ${ }^{11}$ they point to the importance of re-TUR in the management of NMIBC especially in T1 and/or HG tumours.

It is worth mentioning that the presence of residual tumour on re-TUR was associated with higher risks of tumour recurrence and progression compared to patients with T0 on reTUR. We found that $83 \%$ of patients with residual tumour on re-TUR developed recurrence compared to $39 \%$ of patients with T0 $(p<0.001)$. Furthermore, $34 \%$ of patients with residual tumour on re-TUR progressed to muscle-invasive disease compared to $6 \%$ of patients with T0 $(p<0.007)$. Patients with T0 on re-TUR had a significantly longer RFS and PFS compared to patients with residual tumour on reTUR ( $p<0.001, p=0.004$, respectively). Similar results were reported in a previous study in which tumour-free status at repeat TUR was associated with fewer recurrences, longer times to tumour recurrence and less progression. ${ }^{12}$ These findings underscore the importance of complete resection of all visible or suspicious lesions on the outcome of patients with NMIBC.

The major finding in this study is that in multivariate analysis, T stage on re-TUR and the regimen of intra-vesical BCG therapy (induction vs. maintenance) were the only independent prognostic factors for RFS ( $p=0.001, \mathrm{HR}: 1.776)$, $(p<0.001$ HR: 0.165$)$ respectively and PFS $(p=0.014$, HR: 2.114), ( $p=0.008$, HR: 0.097).

In a prospective study including 710 patients with NMIBC, pathology on re-staging TUR and response to treatment at first follow-up cystoscopy were the only independent predicting variables for first tumour recurrence and they were also the most significant predicting factors for tumour progression. ${ }^{8}$ It should be noted that maintenance intravesical BCG therapy was not allowed in this study. A recently published study reported that maintenance intravesical BCG 
Table 3. Multivariate analysis of predictive factors for RFS and PFS

\begin{tabular}{|c|c|c|c|c|c|}
\hline \multirow[b]{2}{*}{ Variables } & \multirow[b]{2}{*}{ Categories } & \multicolumn{2}{|c|}{ RFS } & \multicolumn{2}{|c|}{ PFS } \\
\hline & & $\begin{array}{l}\text { Hazard ratio* } \\
(95 \% \mathrm{Cl})\end{array}$ & $p$ value & $\begin{array}{l}\text { Hazard ratio* } \\
(95 \% \mathrm{Cl})\end{array}$ & $p$ value \\
\hline Hydronephrosis & Yes vs. No & $\begin{array}{c}2.406 \\
(0.733-7.896)\end{array}$ & 0.148 & $\begin{array}{c}1.419 \\
(0.305-6.599)\end{array}$ & 0.656 \\
\hline Multifocality of tumours & Yes vs. No & $\begin{array}{c}0.957 \\
(0.436-2.097)\end{array}$ & 0.912 & $\mathrm{~N} / \mathrm{A}$ & N/A \\
\hline T stage on initial TUR & $\mathrm{Ta}, \mathrm{T} 1$ & N/A & $\mathrm{N} / \mathrm{A}$ & $\begin{array}{c}2.461 \\
(0.820-7.385)\end{array}$ & 0.108 \\
\hline T stage on re-TUR & T0, Ta, CIS, T1 & $\begin{array}{c}1.776 \\
(1.279-2.464)\end{array}$ & 0.001 & $\begin{array}{c}2.114 \\
(1.163-3.845)\end{array}$ & 0.014 \\
\hline $\begin{array}{l}\text { Immediate intravesical } \\
\text { chemotherapy }\end{array}$ & Yes vs. No & $\begin{array}{c}0.854 \\
(0.370-1.970)\end{array}$ & 0.711 & N/A & N/A \\
\hline $\begin{array}{l}\text { Regimen of intravesical BCG } \\
\text { therapy }\end{array}$ & $\begin{array}{l}\text { Induction alone vs. } \\
\text { maintenance }\end{array}$ & $\begin{array}{c}0.165 \\
(0.064-0.424)\end{array}$ & $<0.001$ & $\begin{array}{c}0.097 \\
(0.017-0.542)\end{array}$ & 0.008 \\
\hline
\end{tabular}

*Hazard ratio according to Cox regression analysis. TUR: transurethral resection; RFS: recurrence-free survival; PFS: progression-free survival; BCG: bacillus Calmette-Guérin.

therapy in patients with high recurrence or progression risk improved the rates of 5 -year RFS and PFS compared to induction therapy only $(100 \%$ vs. $18 \%, p=0.009)(100 \%$ vs. $69 \%, p=0.047$ ) respectively. ${ }^{13}$ The limitations of our study are its retrospective nature and the relatively small number of patients in the cohort.

\section{Fig 1.a}

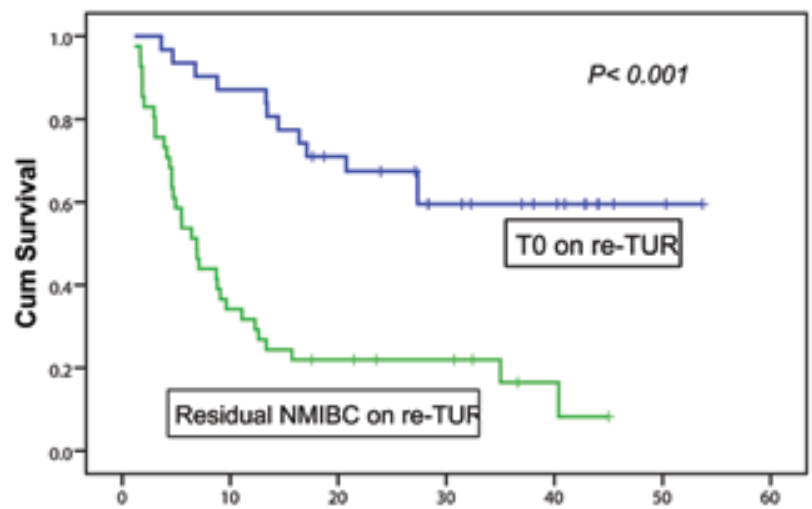

$1 . \mathrm{b}$

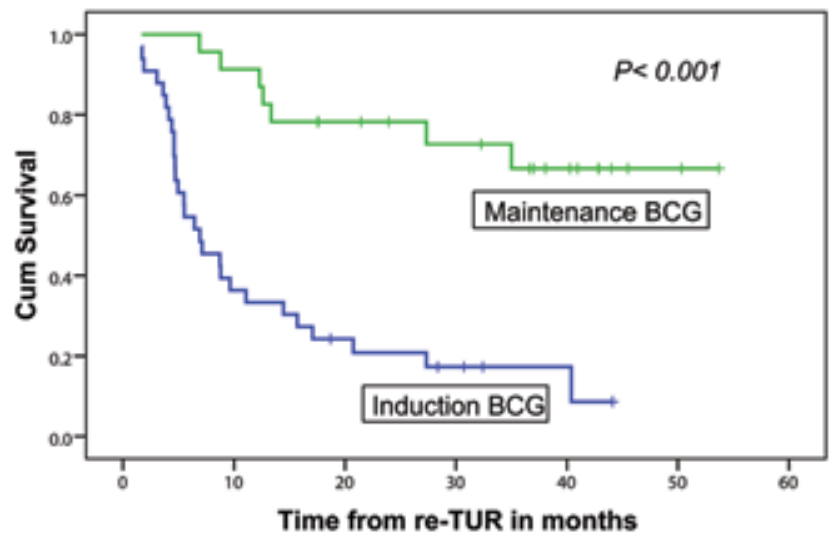

1.c

Progression-free survival

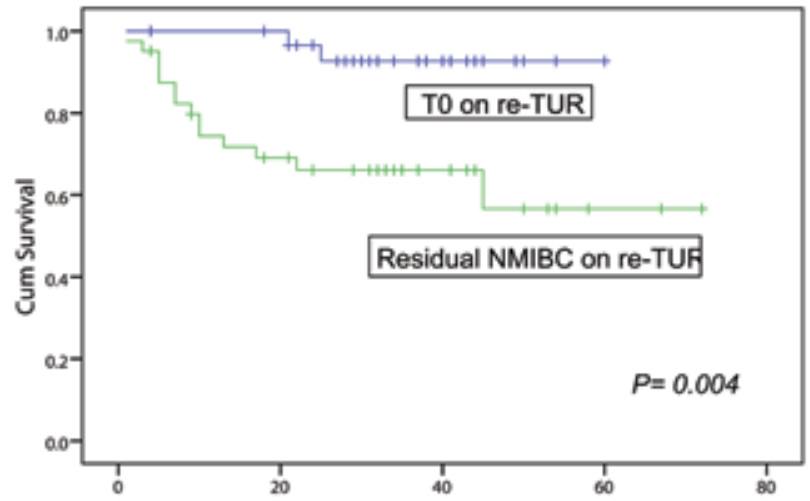

1.d

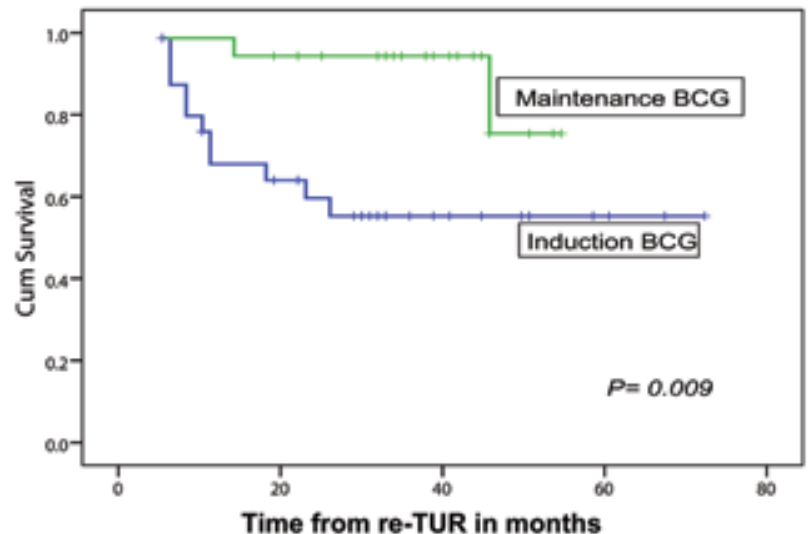

Fig. 1. Kaplan-Meier curves of recurrence-free survival and progression-free survival. A and C: T0 vs. residual non-muscle invasive bladder cancer on retransurethral resection (log-rank test result: 21.545, 8.337, respectively). B and D: Regimen of bacillus Calmette-Guérin (BCG) therapy (induction vs. maintenance) (log-rank test result: 20.885, 6.740. respectively). 
Bishr et al.

\section{Conclusions}

The presence of T0 on re-TUR is associated with a favourable RFS and PFS and could be useful in selecting patients for bladder preservation management. The presence of residual tumour on re-staging TUR requires close followup and in some case early cystectomy should be considered. Maintenance intra-vesical BCG therapy was found to improve the RFS and PFS in patient with high risk NMIBC.

Competing interests: Dr. Bishr and Dr. Latour all declare no competing financial or personal interests. Dr. Saad and Dr. Lattouf are Advisory Board members for Amgen, Astellas, Janssen, Abbott, Sanofi and Bayer. They have also received research grants and honoraria from Amgen, Astellas, Janssen, Abbott, Sanofi and Bayer. They have also participated in clinical trials in the past 2 years for Amgen, Astellas, Janssen, Sanofi and Bayer.

This paper has been peer-reviewed.

\section{References}

1. Herr HW, Sogani PC. Does early cystectomy improve the survival of patients with high risk superficial bladder tumors? J Urol 2001;166:1296-9. http://dx.doi.org/10.1016/S0022-5347(05)65756-4

2. Kulkarni GS, Hakenberg OW, Gschwend JE, et al. An updated critical analysis of the treatment strategy for newly diagnosed high-grade TI (previously TIG3) bladder cancer. Eur Urol 2010;57:60-70. http:// dx.doi.org/10.1016/i.eururo.2009.08.024

3. Herr HW. Role of re-resection in non-muscle-invasive bladder cancer. ScientificWorldJournal 201 1;11:2838. http://dx.doi.org/10.1100/tsw.2011.29
4. Nieder AM, Brausi M, Lamm D, et al. Management of stage $\mathrm{Tl}$ tumors of the bladder: International Consensus Panel. Urology 2005;66:108-25. hittp://dx.doi.org/10.1016/i.urology.2005.08.066

5. Sylvester RJ, van der Meijden AP, Oosterlinck W, et al. Predicting recurrence and progression in individual patients with stage Ta TI bladder cancer using EORTC risk tables: A combined analysis of 2596 patients from seven EORTC trials. Eur Urol 2006;49:466-5; discussion 475-7.

6. Divrik RT, Sahin A, Altok $M$, et al. The frequency of hydronephrosis at initial diagnosis and its effect on recurrence and progression in patients with superficial bladder cancer. J Urol 2007; 178:802-6; discussion 806. http://dx.doi.org/10.1016/i.juro.2007.05.054

7. Orsola A, Trias I, Raventos $C X$, et al. Initial high-grade Tl urothelial cell carcinoma: Feasibility and prognostic significance of lamina propria invasion microstaging (Tla/b/c) in BCG-treated and BCG-non-treated patients. Eur Urol 2005;48:231-8; discussion 238. http://dx.doi.org/10.1016/j.eururo.2005.04.013

8. Herr HW, Donat SM. A re-staging transurethral resection predicts early progression of superficial bladder cancer. BJU Int 2006;97:1194-8. http://dx.doi.org/10.1111/j.1464-410X.2006.06145.x

9. Raj GV, Herr H, Serio AM, et al. Treatment paradigm shift may improve survival of patients with high risk superficial bladder cancer. J Urol 2007;177:1283-6; discussion 1286. http://dx.doi.org/10.1016/i. juro.2006.11.090

10. Huguet J, Crego M, Sabate $S$, et al. Cystectomy in patients with high risk superficial bladder tumors who fail intravesical BCG therapy: Pre-cystectomy prostate involvement as a prognostic factor. Eur Urol 2005;48:53-9; discussion 59. http://dx.doi.org/10.1016/i.eururo.2005.03.021

11. Miladi $M$, Peyromaure $M$, Zerbib $M$, et al. The value of a second transurethral resection in evaluating patients with bladder tumours. Eur Urol 2003;43:241-5. http://dx.doi.org/10.1016/S03022838(03)00040-X

12. Guevara A, Salomon L, Allory Y, et al. The role of tumor-free status in repeat resection before intravesical bacillus Calmette-Guerin for high grade Ta, T1 and CIS bladder cancer. J Urol 2010;183:2161-4. http:// dx.doi.org/10.1016/i.juro.2010.02.026

13. Muto S, Nakaijma A, Horiuchi A, et al. Maintenance therapy with intravesical bacillus Calmette-Guerin in patients with intermediate- or high-risk non-muscle-invasive bladder cancer. Jpn J Clin Oncol 2013;43:30513. http://dx.doi.org/10.1093/iico/hys225

Correspondence: Dr. Fred Saad, Professor and Chief of Urology, Centre Hospitalier de I'Université de Montréal (CHUM), 1560 rue Sherbrooke E., Montreal, QC H2L 4M1; fax: 514-412-7620; fredsaad@videotron.ca 